\title{
FRECUENCIA DE SÍNDROME METABÓLICO EN RESIDENTES DE UNA REGIÓN ANDINA DEL PERÚ
}

\author{
Juana Aurelia Ninatanta-Ortizi,a, Luz Amparo Núñez-Zambrano ${ }^{1, b}$, Segunda Aydeé García-Flores $^{1, c}$, \\ Franco Romaní Romaní ${ }^{2, d}$
}

\begin{abstract}
RESUMEN
Objetivos. Estimar la frecuencia del síndrome metabólico (SM) y sus características asociadas en poblaciones seleccionadas residentes de zonas urbanas de dos distritos de la región Cajamarca. Materiales y métodos. Estudio transversal realizado el 2014, en muestras aleatorias de tres poblaciones de estudio: estudiantes de secundaria, universitarios y madres de estudiantes de primaria. En adultos, el SM se definió mediante los criterios del Third Adult Treatment Panel (ATP III), en adolescentes se usó los criterios modificados de Cook. Se aplicó un cuestionario estructurado sobre prácticas saludables y hábitos nocivos. Se realizaron las estimaciones de SM para cada población de estudio, y estratificadas por sexo. Se realizó el análisis bivariado para identificar características asociadas a SM. Resultados. Enrolamos 1427 participantes (586 fueron escolares de secundaria, 305 universitarios y 536 madres de escolares de primaria). La frecuencia estimada de SM en estudiantes de secundaria fue 3,2\% (IC95\%: 1,7-4,8), 1,6\% (IC95\%: 0,5-3,8) en universitarios y 23,5\% (IC95\%: 19,8 - 27,2) en madres. Los componentes más prevalentes fueron bajo nivel de colesterol HDL $(37,0 \%, 60,5 \%$ y $72,4 \%)$ y la hipertrigliceridemia $(46,4 \%, 29,9 \%$ y $38,4 \%)$, en escolares de secundaria, universitarios y madres respectivamente. Conclusiones. La frecuencia de SM fue mayor en la población de madres de estudiantes de primaria (mujeres adultas). El perfil fenotípico de SM en mujeres adultas se caracterizó por una elevada frecuencia de obesidad abdominal y dislipidemias (hipertrigliceridemia y colesterol HDL bajo) mientras que en adolescentes y universitarios fueron predominantes las dislipidemias.
\end{abstract}

Palabras clave: Síndrome X metabólico; Estudiantes; Madres; Perú (Fuente: DeCS BIREME).

\section{FREQUENCY OF METABOLIC SYNDROME IN RESIDENTS OF AN ANDEAN REGION IN PERU}

\begin{abstract}
Objectives. To calculate the frequency of metabolic syndrome (MetS) and its associated characteristics in selected populations residing in the urban areas of two districts in the Cajamarca region. Materials and methods. Cross-sectional study performed in 2014, obtaining randomized samples from three target study populations: high school students, university students, and schoolchildren mothers. In adults, MetS was defined by means of Third Adult Treatment Panel (ATP III) criteria, and in teenagers, the modified Cook criteria were used. A structured survey on healthy practices and unhealthy habits was implemented. MetS estimations were carried out for each study population, and stratified by sex. A bivariate analysis was performed to identify MetS-related characteristics. Results. We enrolled 1,427 participants (586 high school students, 305 university students, and 536 schoolchildren mothers) The estimated frequency of MetS in high school students was $3.2 \%(95 \%$ confidence interval $[\mathrm{Cl}]=1.7-4.8 \%), 1.6 \%(95 \% \mathrm{Cl}=0.5-3.8 \%)$ in university students, and $23.5 \%(95 \% \mathrm{Cl}=19.8-27.2 \%)$ in mothers. The most prevalent components were low HDL levels $(37.0 \%, 60.5 \%$, and $72.4 \%)$ and hypertriglyceridemia $(46.4 \%, 29.9 \%$ and $38.4 \%)$, in high school students, university students, and mothers, respectively. Conclusions. MetS frequency was higher in the mothers of schoolchildren (adult women). The MetS phenotype profile in adult women was characterized by an elevated frequency of abdominal obesity and dyslipidemia (hypertriglyceridemia and low HDL cholesterol), while dyslipidemia was prevalent in teenagers and university students.
\end{abstract}

Key words: Metabolic syndrome X; Students, Mothers; Peru (Source: MeSH NLM).

\footnotetext{
Escuela Académico Profesional de Enfermería, Facultad Ciencias de la Salud, Universidad Nacional de Cajamarca. Cajamarca, Perú

Oficina General de Investigación y Transferencia Tecnológica, Instituto Nacional de Salud. Lima, Perú

a Enfermera, doctora en Ciencias de Enfermería, ${ }^{\mathrm{b}}$ enfermera, magíster en Promoción y Educación para la Salud, ${ }^{\mathrm{c}}$ enfermera, magíster en Salud Pública, ¿ médico cirujano

Recibido: 11/06/2016 Aprobado: 12/10/2016
}

Citar como: Ninatanta-Ortiz JA, Núñez-Zambrano LA, García-Flores SA, Romaní Romaní F. Frecuencia de síndrome metabólico en residentes de una región andina del Perú. Rev Peru Med Exp Salud Publica. 2016;33(4):640-50. doi: 10.17843/rpmesp.2016.334.2546 


\section{INTRODUCCIÓN}

El síndrome metabólico (SM) es un constructo de cinco factores de riesgo para enfermedad cardiovascular ateroesclerótica y diabetes mellitus tipo 2 (1). Su prevalencia en América Latina ajustada por edad y sexo se estima en $29,5 \%$, dicha estimación realizada el 2015 por un metaanálisis incluyó solo estudios transversales que usaron los criterios diagnósticos del Adult Treatment Panel (ATP III) (2). En el Perú, el estudio PREVENCION realizado en la ciudad de Arequipa (2004 y 2006) reporta una prevalencia de $18,1 \%$ (criterios ATP III) ${ }^{(3)}$. Otro estudio realizado el 2011, en estudiantes de secundaria, universitarios y madres de estudiantes de primaria de las ciudades de Trujillo, Arequipa, Lima y Callao, estimó prevalencias de algunos componentes del SM, sin realizar una estimación global de su ocurrencia. Entre dichas estimaciones destaca la elevada proporción de participantes con niveles bajos de colesterol HDL $(\mathrm{C}-\mathrm{HDL})^{(4)}$.

El patrón fenotípico del SM en adultos residentes en zonas andinas de Sudamérica se caracteriza por alta prevalencia de obesidad abdominal, incremento de la prevalencia de SM a mayor grupo etario, y alta prevalencia de dislipidemias, específicamente bajos niveles de C-HDL en mujeres e hipertrigliceridemia en varones ${ }^{(5)}$. Este perfil se desprende de estudios transversales realizados en muestras representativas de adultos mestizos de zonas andinas de Venezuela, Perú, Chile, Ecuador y Colombia, los cuales han estimado prevalencias de SM que varían de 14,0 a $31,6 \%{ }^{(2,3,5,6)}$.

La etnicidad es un determinante importante en el fenotipo del SM, debido, probablemente, a factores genéticos, patrones de la dieta y niveles de actividad física ${ }^{(5,7)}$. En los andes peruanos, especialmente en zonas urbanas, los individuos en diversos escenarios (hogar, escuelas, centros de formación y laborales) están expuestas a la occidentalización de la dieta, formas de vida sedentarias, y otros cambios culturales ${ }^{(5)}$. En este contexto, y frente a la limitada evidencia ${ }^{(8,9,10)}$ sobre la magnitud, perfil fenotípico y características asociadas al SM; el presente estudio busca contribuir con la detección temprana y posible prevención del SM mediante la caracterización fenotípica en adolescentes y adultos jóvenes residentes de zonas alto andinas.

Estudios realizados en zonas andinas muestran que el SM se incrementa a mayor edad, especialmente en mujeres ${ }^{(3,5)}$. También existe evidencia de la importancia de la detección del SM en mujeres, pues se ha encontrado asociación entre la presencia de SM en madres y su descendencia femenina, esta asociación se presenta, incluso, para algunos componentes aislados del SM, como glucosa elevada, obesidad central y niveles bajos de C-HDL (11). También se ha reportado asociación entre obesidad pregestacional con mayor índice de masa corporal, mayores niveles de presión arterial, y resistencia a la insulina en la descendencia $(11,12)$. Estos hallazgos sugieren la presencia de mecanismos intrauterinos en la aparición del SM, así como el rol de los estilos de vida y del entorno compartido entre las madres e hijos.

En el Perú, el Ministerio de Salud (MINSA) ha diseñado programas de promoción de la salud que consideran entre sus grupos objetivos a los niños, adolescentes y adultos, en sus diferentes escenarios de vida, como son los centros laborales, viviendas, e instituciones educativas (13). El 2016, el MINSA estableció Lineamientos de Políticas y Estrategias para la Prevención y Control de Enfermedades No Transmisibles (ENT), entre ellos se plantea para población general, así como para poblaciones en riesgo, el incremento de factores protectores y la reducción de factores de riesgos modificables de las ENT (14). Por lo expuesto, el presente estudio busca estimar la frecuencia de $\mathrm{SM}$ en tres poblaciones priorizadas (adolescentes, universitarios, y madres de estudiantes de primaria) en zonas urbanas andinas, además, busca identificar características asociadas al SM para dichas poblaciones. Los resultados en estas tres poblaciones específicas captadas en escenarios puntuales, brindarán evidencias para focalizar intervenciones dirigidas a reducir la ocurrencia del SM, así como de sus componentes, en escuelas y centros universitarios.

\section{MATERIALES Y MÉTODOS}

\section{DISEÑO Y POBLACIÓN DE ESTUDIO}

La región Cajamarca está ubicada en la zona norte del Perú. El estudio fue realizado en los distritos de Chota (2388 m de altitud) y Cajamarca (2750 m de altitud). El distrito de Cajamarca, ubicado en la provincia del mismo nombre, cuenta con 246536 habitantes (2015), y un porcentaje de pobreza del $29,8 \%$. El distrito de Chota, ubicado en la provincia de Chota, cuenta con 48698 habitantes (2015) y un porcentaje de pobreza del $36,0 \%$ (Series Nacionales Instituto Nacional de Estadística e Informática).

Realizamos un estudio transversal en tres poblaciones. La población de estudiantes del primer y segundo grado de secundaria provino de cuatro escuelas del distrito de Cajamarca y una del distrito de Chota. Las escuelas fueron seleccionadas por ser los centros educativos con mayor alumnado en el nivel secundario y primario en dichos ámbitos. Los universitarios fueron estudiantes del primer y segundo año de las facultades de la Universidad Nacional de Cajamarca (UNC). La UNC es la principal 
universidad pública de la región y concentra la mayor cantidad de universitarios. Se abordó solo estudiantes (educación secundaria y universitaria) y madres de los dos primeros años de estudio, pues concentran el grupo etario de interés para el estudio, y permite tener una línea basal del SM para evaluar el impacto de intervenciones futuras en dichos ámbitos de estudio.

El tamaño muestral se estimó considerando un tamaño poblacional conocido. Se consideró una prevalencia de SM de 19,1\% para escolares de secundaria ${ }^{(12)}, 27,1 \%$ para universitarios ${ }^{(15)}$ y $39,7 \%$ para madres ${ }^{(3)}$; un nivel de confianza del $95 \%$, un margen de error relativo del $15 \%$ para estudiantes de secundaria y universitarios, y del $7 \%$ para madres de primaria. Para estudiantes de secundaria y madres de primaria se utilizó la fórmula para muestreo por conglomerados. El cálculo de tamaño muestral fue realizado para cada escuela en forma independiente, el tamaño muestral total estuvo dado por la sumatoria del tamaño muestral planeado para cada escuela. El diseño muestral empleado permitió realizar estimaciones de SM para cada escuela; y aseguró un tamaño muestral representativo para la población de estudio ${ }^{(4)}$. En universitarios se utilizó la fórmula de muestreo aleatorio estratificado proporcional al tamaño del estrato. Con dichos supuestos el tamaño muestral fue de 575 estudiantes de secundaria, 387 universitarios y 559 madres de estudiantes de primaria.

El trabajo de campo en estudiantes de secundaria y madres de primaria fue realizado entre junio y octubre, y en universitarios entre octubre y diciembre de 2014 . La selección de estudiantes de secundaria y madres de primaria fue mediante un muestreo por conglomerados monoetápico (se consideró como conglomerado a las secciones/aulas) sin submuestreo. Los universitarios fueron seleccionados mediante muestreo aleatorio estratificado, considerando como estrato las carreras universitarias. Los criterios de exclusión para los tres grupos fueron: gestación, discapacidad física que imposibilite la medición antropométrica, no aceptar participar del estudio, ausentes por descanso médico o inasistencia. Para el caso de universitarios, si el participante seleccionado no fue encontrado durante el enrolamiento, o no aceptó participar del estudio, se invitó al inmediato superior (en primera instancia) o al inferior del marco muestral.

\section{MEDICIÓN DE VARIABLES}

En adultos se usó la definición de SM del National Cholesterol Education Program, Third Adult Treatment Panel (NCEP ATP III) ${ }^{(16,17)}$, la cual determina SM con la presencia de tres o más de cinco factores de riesgo: glucosa plasmática en ayunas $\geq 110 \mathrm{md} /$ $\mathrm{dL}$ o tratamiento farmacológico para hiperglicemia, obesidad central definida como circunferencia de cintura $>102$ centímetros $(\mathrm{cm})$ en varones y $>88 \mathrm{~cm}$ en mujeres, presión arterial $\geq 130 / 85 \mathrm{mmHg}$ o tratamiento antihipertensivo en paciente con historia de hipertensión, triglicéridos $\geq 150 \mathrm{mg} / \mathrm{dL}$ o tratamiento farmacológico para hipertrigliceridemia; y colesterol HDL (C-HDL) $<40$ $\mathrm{mg} / \mathrm{dL}$ en varones $\mathrm{y}<50 \mathrm{mg} / \mathrm{dL}$ en mujeres o tratamiento farmacológico para reducir C-HDL.

En adolescentes (12 a <18 años) se usó los criterios modificados de Cook ${ }^{(18)}$, que define SM con la presencia de tres o más de cinco factores: circunferencia de cintura $\geq$ percentil 90 para edad y sexo, glucosa plasmática en ayunas $\geq 110 \mathrm{md} / \mathrm{dL}$, presión arterial sistólica o diastólica $\geq$ percentil 90 para edad/sexo/talla o tratamiento antihipertensivo, triglicéridos $\geq 110 \mathrm{mg} / \mathrm{dL}$, y $\mathrm{C}-\mathrm{HDL} \leq 40 \mathrm{mg} / \mathrm{dL}$. Se usaron los criterios de Cook, debido a que usa percentiles para definir dos de sus cinco componentes (obesidad abdominal y presión arterial), de esta forma, dicha definición identifica un mayor número de casos de SM comparado con otras definiciones adaptadas para adolescentes ${ }^{(7,19,20)}$.

Se recolectó información sobre antecedentes familiares de diabetes mellitus (DM), hipertensión arterial (HTA) y accidente cerebro vascular (ACV). Dentro de prácticas saludables y hábitos nocivos, se consideraron las siguientes variables: consumo apropiado de agua (ingesta de $\geq 5$ vasos de agua pura/día); descanso óptimo, (siete a ocho horas de sueño/noche); consumo adecuado de frutas y verduras (consumo de $\geq 4$ días por semana); consumo de cigarrillos (sí/no); consumo de alcohol (sí/ no); consumo de bebidas gaseosas, definido como el número de días de consumo por semana (categorizado como "no consumo", "uno a tres días/semana", "cuatro a siete días/semana"), fue recategorizada como dicotómica (sí/no), y consumo de snacks, definido como el número de días de consumo por semana (categorizado como "no consumo", "uno a tres días/semana", "cuatro a siete días/semana"), fue recategorizada como dicotómica (sí/no). Respecto a la actividad física se midió usando el cuestionario internacional de actividad física (IPAQ) versión corta y sus criterios para definir los niveles bajo, moderado y alta ${ }^{(21)}$.

La valoración nutricional de los adultos según índice de masa corporal (IMC) se realizó de acuerdo con la clasificación de Organización Mundial de la Salud (OMS), considerando como sobrepeso el IMC de 25 a $<30 \mathrm{~kg} / \mathrm{m}^{2}$, y obesidad si el IMC fue $\geq 30 \mathrm{~kg} / \mathrm{m}^{2}{ }^{(22)}$. En adolescentes, se usaron los estándares de crecimiento infantil de la OMS-2007 para mayores de cinco años, se clasificó según puntuación z del IMC para la edad (ZIMC) usando el archivo SPSS de la OMS (http://www.who.int/ childgrowth/software/es/). Se consideró como sobrepeso si $Z M I C>+1$ y $\leq+2$ y obesidad si ZMIC $>+2^{(23)}$. 


\section{RECOLECCIÓN DE DATOS}

Se aplicó un cuestionario estructurado sobre datos sociodemográficos, antecedentes familiares patológicos, prácticas saludables y hábitos nocivos. La antropometría (peso, talla y perímetro abdominal) fue realizada por personal de salud certificado por el Centro Nacional de Alimentación y Nutrición (CENAN) del Instituto Nacional de Salud (INS), siguiendo las guías técnicas nacionales de valoración nutricional antropométrica de la persona adulta ${ }^{(22)}$ y del adolescente ${ }^{(23)}$.

La evaluación de presión arterial se realizó con un tensiómetro aneroide (Riester exacta $\left.{ }^{\circledR}\right)$ y estetoscopio (Riester Duplex®). Se consideró el promedio de tres mediciones de presión arterial sistólica y diastólica. La medición en adultos se realizó según norma técnica nacional ${ }^{(24)}$, en adolescentes la medición fue en posición sentado, con reposo de $15 \mathrm{~min}$, el brazo derecho descubierto apoyado en una mesa, flexionado a la altura del corazón, el manguito inflable cubrió dos terceras partes del largo y circunferencia del brazo.

La evaluación bioquímica se realizó con al menos ocho horas de ayuno, se extrajeron muestras sanguíneas por venopunción del antebrazo, se utilizó un tubo de 2 $\mathrm{mL}$ con fluoruro para el dosaje de glucosa y otro tubo de $10 \mathrm{~mL}$ sin anticoagulante para el dosaje de perfil lipídico (colesterol total, colesterol HDL, colesterol LDL y triglicéridos). Las muestras fueron transportadas a un laboratorio particular donde fueron centrifugadas y separadas en alícuotas y congeladas a $-20{ }^{\circ} \mathrm{C}$ hasta su procesamiento en el Laboratorio de Patología Clínica del Centro Nacional de Salud Pública del INS. Los niveles de glucosa, colesterol total y triglicéridos fueron determinados por métodos enzimáticos, el colesterol HDL y LDL por métodos homogéneos, usando reactivos Roche Diagnostic en un equipo automatizado (Cobas C111 analyzer, fabricante Roche Diagnostic Limited). Se verificó la calidad de los resultados usando sueros controles (normal y patológico) comerciales.

\section{ANÁLISIS ESTADÍSTICO}

Se estimó la frecuencia de SM y sus componentes con un intervalo de confianza al 95\%, dicha estimación se realizó para cada población de estudio y estratificado por sexo. Se realizó el análisis descriptivo de las variables sociodemográficas, antecedentes, hábitos nocivos y prácticas saludables usando porcentajes para el caso de las variables cualitativas, y medias y desviación estándar para las cuantitativas. La comparación de medias entre las tres poblaciones de estudio se realizó mediante ANOVA. Se realizó el análisis bivariado usando las pruebas chi cuadrado o exacta de Fisher según corresponda, considerando al SM como variable resultado, además, se estimó la fuerza de asociación mediante el Odds Ratio (OR) crudo. Todas las pruebas estadísticas se realizaron para cada población de estudio. Se consideró un p valor <0,05 como significativo. El análisis estadístico fue realizado en el paquete SPSS versión 22 para Windows (Statistical Package for Social Sciences version 15, Chicago, IL, USA).

\section{ASPECTOS ÉTICOS}

El estudio fue aprobado por el Comité de Ética en Investigación del Instituto Nacional de Salud de Perú. Todos los participantes fueron informados de los objetivos del estudio, los procedimientos se aplicaron previo consentimiento/asentimiento informado escrito.

\section{RESULTADOS}

En el grupo de estudiantes de secundaria se enroló al total de la muestra planeada, en universitarios se enroló el $78,8 \%$ y en madres de primaria al $95,9 \%$. El estudio tuvo una muestra enrolada de 1427 personas, de los cuales $586(41,1 \%)$ fueron escolares de educación secundaria, $305(21,4 \%)$ fueron estudiantes universitarios y 536 $(37,6 \%)$ fueron madres de escolares de primaria.

Del total de estudiantes de secundaria y universitarios, el 52,0 y $49,5 \%$, respectivamente, fueron mujeres. Las medias de edad entre los tres grupos presentan diferencias significativas $(p<0,001)$. La proporción de sobrepeso y obesidad es mayor en el grupo de madres comparado con lo encontrado en escolares y universitarios $(p<0,001)$. Con respecto a la actividad física, una mayor proporción de universitarios $(70,4 \%)$ y madres $(67,2 \%)$ realizan actividad física de baja intensidad, mientras que los estudiantes de secundaria realizan en mayor proporción actividad física de alta intensidad $(p=0,002)$ (Tabla 1).

Respecto a las variables bioquímicas, la media del colesterol total es mayor en madres, comparada con universitarios y estudiantes de secundaria $(p<0,001)$. En estudiantes de secundaria la media de colesterol total es mayor en mujeres $(160,0 \pm 29,2$ versus $153,1 \pm 27,2$, $p=0,003)$, al igual que en universitarios (171,3 $\pm 35,2$ versus $163,3 \pm 31,1, p=0,038)$. Respecto al colesterol LDL, la media es mayor en el grupo de madres de primaria $(p<0,001)$. En estudiantes de secundaria, la media de colesterol LDL es mayor en mujeres $(95,6$ $\pm 23,4$ versus $90,6 \pm 23,2, p=0,009)$, en estudiantes universitarios no se encontró diferencias significativas $(103,9 \pm 28,5$ versus $98,2 \pm 24,9, p=0,059)$. La media de triglicéridos es mayor en el grupo de madres de educación primaria $(p<0,001)$ (Tabla 2).

\section{FRECUENCIA DE SÍNDROME METABÓLICO}

La frecuencia de SM en madres de estudiantes de primaria fue de 23,5\% (IC95\%: 19,8-27,2), en estudiantes de secundaria fue de 3,2\% (IC95\%: 1,74,8 ) y en universitarios de 1,6\% (IC95\%: 0,5-3,8\%). Considerando solo mujeres, la frecuencia de SM se 
Tabla 1. Características generales de estudiantes de educación secundaria, universitarios y madres de estudiantes de primaria, región Cajamarca, Perú 2014

\begin{tabular}{|c|c|c|c|c|}
\hline Características & $\begin{array}{c}\text { Estudiantes } \\
\text { educación } \\
\text { secundaria } \\
n=586\end{array}$ & $\begin{array}{c}\text { Estudiantes } \\
\text { universitarios } \\
n=305\end{array}$ & $\begin{array}{c}\text { Madres de } \\
\text { estudiantes de } \\
\text { primaria } \\
n=536\end{array}$ & ${ }^{*}$ Valor $p$ \\
\hline & $n(\%)$ & $n(\%)$ & $n(\%)$ & \\
\hline Edad (años \pm DE) & $12,74 \pm 0,94$ & $20,26 \pm 2,63$ & $34,24 \pm 8,08$ & $<0,001^{\dagger}$ \\
\hline Antecedente familiar de DM & $42(7,2)$ & $32(10,5)$ & $33(6,2)$ & 0,066 \\
\hline Antecedente familiar de HTA & $80(13,7)$ & $60(19,7)$ & $153(28,5)$ & $<0,001$ \\
\hline Antecedente familiar de ACV & $15(2,6)$ & $23(7,5)$ & $33(6,2)$ & 0,001 \\
\hline Consumo de cigarrillos (Si) & $59(10,1)$ & $141(46,2)$ & $60(11,2)$ & $<0,001$ \\
\hline Consumo de alcohol (Si) & $261(44,5)$ & $271(88,9)$ & $427(80,1)$ & $<0,001$ \\
\hline Consumo de gaseosas & & & & $<0,001$ \\
\hline No consume & $110(18,8)$ & $114(37,4)$ & $204(38,1)$ & \\
\hline 1 a 3 días/sem & $413(70,5)$ & $167(54,8)$ & $299(55,8)$ & \\
\hline 4 a 7 días/sem & $63(10,8)$ & $24(7,9)$ & $33(6,2)$ & \\
\hline Consumo de snacks & & & & $<0,001$ \\
\hline No consume & $52(8,9)$ & $75(24,6)$ & $294(54,9)$ & \\
\hline 1 a 3 días/sem & $366(62,6)$ & $182(59,7)$ & $228(42,5)$ & \\
\hline 4 a 7 días/sem & $167(28,5)$ & $48(15,7)$ & $14(2,6)$ & \\
\hline $\begin{array}{l}\text { Consumo apropiado de agua pura } \\
\text { ( } \geq 5 \text { vasos/día) }\end{array}$ & $105(17,9)$ & $68(22,3)$ & $86(16,0)$ & 0,076 \\
\hline Horas de sueño por día (óptimo) & $349(59,6)$ & $153(50,2)$ & $367(68,5)$ & $<0,001$ \\
\hline Consumo de frutas & & & & 0,095 \\
\hline No consume & $9(1,5)$ & $4(1,3)$ & $6(1,1)$ & \\
\hline 1 a 3 días/sem & $171(29,2)$ & $111(36,5)$ & $193(36,1)$ & \\
\hline 4 a 7 días/sem & $406(69,3)$ & $189(62,2)$ & $336(62,8)$ & \\
\hline Consumo de verduras & & & & $<0,001$ \\
\hline No consume & $58(9,9)$ & $16(5,3)$ & $7(1,3)$ & \\
\hline 1 a 3 días/sem & $313(53,4)$ & $142(46,7)$ & $213(39,7)$ & \\
\hline 4 a 7 días/sem & $215(36,7)$ & $146(48,0)$ & $316(59,0)$ & \\
\hline Seguro de Salud (Si) & $437(75,0)$ & $238(78,3)$ & $372(69,5)$ & 0,014 \\
\hline Estado nutricional & & & & $<0,001$ \\
\hline No sobrepeso, ni obesidad & $412(70,3)$ & $223(73,1)$ & $147(27,4)$ & \\
\hline Sobrepeso & $134(22,9)$ & $71(23,3)$ & $263(49,1)$ & \\
\hline Obesidad & $40(6,8)$ & $11(3,6)$ & $126(23,5)$ & \\
\hline Intensidad de actividad físicał & & & & 0,002 \\
\hline Baja & $373(63,9)$ & $214(70,4)$ & $359(67,2)$ & \\
\hline Moderada & $119(20,4)$ & $56(18,4)$ & $129(24,2)$ & \\
\hline Alta & $92(15,8)$ & $34(11,2)$ & $46(8,6)$ & \\
\hline
\end{tabular}

DM: diabetes mellitus, HTA: hipertensión arterial, ACV: accidente cerebrovascular

${ }^{*}$ Chi cuadrado, ${ }^{\dagger}$ Prueba de ANOVA

‡Datos perdidos: dos en estudiantes de secundaria, dos en madres de estudiantes de primaria y uno en universitarios

incrementa de 2,3 , a $3,3 \%$ y $23,5 \%$, en estudiantes de secundaria, universitarias y madres de primaria, respectivamente. En varones universitarios no se identificaron casos de SM.

Los componentes de SM más frecuentes fueron la hipertrigliceridemia y el bajo nivel de C-HDL. En estudiantes de secundaria la frecuencia de niveles bajos de C-HDL fue de $37,0 \%$, frecuencias mayores se encontraron en universitarios $(60,5 \%)$ y en madres de primaria $(72,4 \%)$. No se identificaron casos de estudiantes de secundaria y universitarios del sexo femenino que presentaran glucosa plasmática en ayunas $\geq 100 \mathrm{mg} / \mathrm{dL}$ (Tabla 3 ).

La obesidad abdominal se encontró en el53,6\% de madres de estudiantes de primaria, mientras que en estudiantes universitarios y de secundaria dicha proporción fue de 2,9 y $5,6 \%$ respectivamente. La frecuencia de HTA fue 
Tabla 2. Variables bioquímicas en estudiantes de educación secundaria, universitarios y madres de estudiantes de primaria, región Cajamarca, Perú 2014

\begin{tabular}{|c|c|c|c|c|}
\hline Variables bioquímicas & $\begin{array}{c}\text { Estudiantes } \\
\text { educación } \\
\text { secundaria }\end{array}$ & $\begin{array}{c}\text { Estudiantes } \\
\text { universitarios }\end{array}$ & $\begin{array}{c}\text { Madres de estudiantes } \\
\text { de primaria }\end{array}$ & \\
\hline & Media ( $\pm D E)$ & Media ( $\pm D E)$ & Media ( $\pm D E)$ & ${ }^{*}$ Valor $p$ \\
\hline Glucosa en ayunas (mg/dL) & $90,5(6,1)$ & $86,9(6,9)$ & $88,3(12,3)$ & $<0,001$ \\
\hline Colesterol total $(\mathrm{md} / \mathrm{dL})$ & $156,7(28,4)$ & $167,3(33,5)$ & $187,9(39,5)$ & $<0,001$ \\
\hline Colesterol HDL (md/dL) & $44,7(11,5)$ & $42,5(9,9)$ & $43,7(11,4)$ & 0,019 \\
\hline Colesterol LDL (md/dL) & $93,2(23,4)$ & $101,0(26,9)$ & $118,8(32,5)$ & $<0,001$ \\
\hline Triglicéridos (md/dL) & $118,3(56,6)$ & $131,0(68,2)$ & $150,7(81,2)$ & $<0,001$ \\
\hline
\end{tabular}

${ }^{*}$ Prueba de ANOVA

de $4,7 \%$ en madres de estudiantes de primaria y solo se presentó en 0,8 y $0,7 \%$ de estudiantes de secundaria y universitarios, respectivamente (Tabla 3).

El $56,8 \%$ de estudiantes de secundaria, $65,9 \%$ de universitarios y $63,4 \%$ de madres, presentaron solo uno o dos componentes de SM de forma aislada. El $3,1 \%$ de los estudiantes de secundaria presentaron tres componentes de SM y $0,2 \%$ cuatro componentes. En el grupo de universitarios, 1,6\% presentaron tres componentes; mientras que $23,9 \%$ de madres presentaron tres o más componentes (Tabla 4).

Tabla 3. Prevalencia de síndrome metabólico (SM) y sus componentes en estudiantes de educación secundaria, universitarios y madres de estudiantes de primaria, región Cajamarca, Perú 2014

\begin{tabular}{|c|c|c|c|}
\hline \multirow[t]{2}{*}{ Componentes } & $\begin{array}{c}\begin{array}{c}\text { Estudiantes educación } \\
\text { secundaria } \\
n=586\end{array} \\
\end{array}$ & $\begin{array}{l}\text { Estudiantes universitarios } \\
\qquad \mathrm{n}=305\end{array}$ & $\begin{array}{c}\text { Madres de estudiantes de } \\
\text { primaria } \\
n=536\end{array}$ \\
\hline & Prevalencia (IC95\%) & Prevalencia (IC95\%) & Prevalencia (IC95\%) \\
\hline \multicolumn{4}{|c|}{ Síndrome metabólico } \\
\hline Total & $3,2(1,7-4,8)$ & $1,6(0,5-3,8)$ & $23,5(19,8-27,2)$ \\
\hline Hombres & $4,3(1,7-6,8)$ & $0^{*}$ & - \\
\hline Mujeres & $2,3(0,5-4,1)$ & $3,3(1,1-7,6)$ & $23,5(19,8-27,2)$ \\
\hline \multicolumn{4}{|c|}{$\begin{array}{l}\text { Glucosa plasmática en ayunas } \\
\text { elevada }\end{array}$} \\
\hline Total & $0,3(0,04-1,2)$ & $0,7(0,1-2,4)$ & $1,5(0,4-2,6)$ \\
\hline Hombres & $0,7(0,1-2,6)$ & $1,3(0,2-4,6)$ & - \\
\hline Mujeres & $0^{*}$ & $0^{*}$ & $1,5(0,4-2,6)$ \\
\hline \multicolumn{4}{|c|}{ Obesidad abdominal } \\
\hline Total & $5,6(3,7-7,6)$ & $2,9(0,9-5,0)$ & $53,6(49,2-57,9)$ \\
\hline Hombres & $7,5(4,2-10,7)$ & $0,7(0,02-3,6)$ & - \\
\hline Mujeres & $3,9(1,6-6,3)$ & $5,3(1,4-9,2)$ & $53,6(49,2-57,9)$ \\
\hline \multicolumn{4}{|c|}{ Hipertensión arterial } \\
\hline Total & $0,8(0,3-1,9)$ & $0,7(0,1-2,4)$ & $4,7(2,8-6,6)$ \\
\hline Hombres & $1,1(0,2-3,1)$ & $0,7(0,02-3,6)$ & - \\
\hline Mujeres & $0,7(0,1-2,4)$ & $0,7(0,02-3,6)$ & $4,7(2,8-6,6)$ \\
\hline \multicolumn{4}{|c|}{ Hipertrigliceridemia } \\
\hline Total & $46,4(42,3-50,5)$ & $29,9(24,6-35,3)$ & $38,4(34,2-42,6)$ \\
\hline Hombres & $43,8(37,8-49,8)$ & $31,2(23,5-38,8)$ & - \\
\hline Mujeres & $48,9(43,1-54,6)$ & $28,7(21,1-36,2)$ & $38,4(34,2-42,6)$ \\
\hline \multicolumn{4}{|c|}{ Colesterol HDL bajo } \\
\hline Total & $37,0(33,0-41,0)$ & $60,5(54,9-66,2)$ & $72,4(68,5-76,3)$ \\
\hline Hombres & $37,0(31,2-42,8)$ & $47,4(39,2-55,6)$ & - \\
\hline Mujeres & $37,1(31,5-42,6)$ & $74,0(66,7-81,4)$ & $72,4(68,5-76,3)$ \\
\hline
\end{tabular}

IC95\%: intervalo de confianza del 95\%

${ }^{*}$ No se encontraron casos que cumplieran con los criterios de síndrome metabólico 
Tabla 4. Frecuencia de componentes de síndrome metabólico (SM) en estudiantes de educación secundaria, universitarios y madres de estudiantes de primaria, región Cajamarca, Perú 2014

\begin{tabular}{|c|c|c|c|}
\hline $\begin{array}{l}\text { Número de } \\
\text { componentes } \\
\text { de SM* }\end{array}$ & $\begin{array}{l}\text { Estudiantes } \\
\text { educación } \\
\text { secundaria }\end{array}$ & $\begin{array}{c}\text { Estudiantes } \\
\text { universitarios }\end{array}$ & $\begin{array}{l}\text { Madres de } \\
\text { estudiantes } \\
\text { de primaria }\end{array}$ \\
\hline & n (\%) & n (\%) & n (\%) \\
\hline Ninguno & $234(39,9)$ & $99(32,5)$ & $68(12,7)$ \\
\hline 1 & $195(33,3)$ & $129(42,3)$ & $163(30,4)$ \\
\hline 2 & $138(23,5)$ & $72(23,6)$ & $177(33,0)$ \\
\hline 3 & $18(3,1)$ & $5(1,6)$ & $115(21,5)$ \\
\hline 4 & $1(0,2)$ & $0^{\dagger}$ & $13(2,4)$ \\
\hline Total & 586 & 305 & 536 \\
\hline
\end{tabular}

*No hubo casos que presentaron cinco componentes de síndrome metabólico

+ No se encontraron casos

\section{CARACTERISTICAS ASOCIADAS A SÍNDROME METABÓLICO}

Se analizaron las características asociadas al SM en los tres grupos de estudio por separado (Tabla 5). En estudiantes de secundaria se encontró que el antecedente familiar de diabetes mellitus (OR crudo 3,71, IC95\%: $1,17-11,74)$ y el presentar exceso de peso (OR crudo: 47,42, IC95\%: 6,28-358,24) resultaron asociados al SM. En universitarios, el análisis bivariado muestra que el sexo femenino $(p=0,029)$ y el presentar exceso de peso $(p=0,001)$ fueron características asociadas al SM; para ambas variables no se calculó el OR crudo debido a que una de las celdas presentó un valor observado de cero. En mujeres universitarias la proporción de SM fue de $3,3 \%$ mientras que en varones no se presentaron casos, por otro lado, en universitarios con exceso de peso (incluyendo sobrepeso y obesidad) la proporción de SM fue del $6,1 \%$, mientras que en aquellos sin sobrepeso ni obesidad no se encontraron casos de SM. En madres de primaria, el tener un moderado o alto nivel de actividad física resultó un factor protector para el SM (OR crudo: 0,6, IC95\%: 0,4-0.9), al igual que el consumo adecuado de verduras (OR crudo: 0,57, IC95\%: 0,38-0,85). El exceso de peso también resultó asociado a la presencia de SM (OR crudo: 5,82, IC95\%: 2,96-11,46).

\section{DISCUSIÓN}

En la presente investigación la ocurrencia de SM tuvo diferentes magnitudes según población de estudio. Las madres de estudiantes de primaria fueron las más afectadas con $23,5 \%$, mientras que en estudiantes de secundaria $(3,2 \%)$ y universitarios $(1,6 \%)$ la magnitud fue menor. La frecuencia de SM en madres de primaria es similar al $25,3 \%$ estimado por un metaanálisis de estudios con mujeres adultas latinoamericanas aparentemente sanas ${ }^{(2)}$. Comparando con otras poblaciones andinas peruanas, la frecuencia en madres es similar a lo estimado en Arequipa (2003-2007), que encontró un $23,2 \%$ de mujeres adultas afectadas ${ }^{(3)}$. Para el periodo 2004-2005, la prevalencia de SM (usando criterios ATP III) en mujeres peruanas de $\geq 20$ años residentes por encima de los $3000 \mathrm{~m}$ de altitud fue de 15,2\%; nuestros resultados se aproximan más a la prevalencia estimada en mujeres residentes por debajo de los $1000 \mathrm{~m}$ de altitud $(29,9 \%)^{(25)}$. Las frecuencias de SM en mujeres de los andes del Perú ${ }^{(3,25)}$, incluyendo nuestros hallazgos, son inferiores a las reportadas en mujeres adultas residentes en los andes de Venezuela $(29,8 \%)$ y Chile $(30,8 \%)^{(5)}$.

El incremento del SM en el grupo de madres comparado con mujeres estudiantes de secundaria y universitarias, se debería al marcado incremento de la frecuencia de obesidad abdominal en madres (53,6\% versus $5,3 \%$ en universitarias y $3,9 \%$ en escolares) y en menor medida a la frecuencia de HTA en madres $(4,7 \%$ en madres versus $0,7 \%$ en universitarias y estudiantes de secundaria mujeres). Esto es consistente con lo encontrado en Arequipa, donde en mujeres de $\geq 50$ años, la frecuencia de SM llegó a $52,8 \%$, ésta alta proporción resultó del pronunciado incremento de la prevalencia de HTA, hipertrigliceridemia y obesidad abdominal en mujeres de dicho grupo etario ${ }^{(3)}$.

La frecuencia de SM en universitarios fue 1,6\%, la totalidad de casos fueron mujeres, en quienes la proporción llegó a 3,3\%. Esta magnitud es inferior a lo reportado en universitarios de Chile $(4,9 \%$ en el $2007)^{(26)}$, Ecuador $(7,5 \% \text { en el } 2015)^{(10)}$, y Brasil $(3,5 \%$ en el 2013) (27). En nuestro estudio, la mayoría de universitarios presentaron uno $(42,3 \%)$ o dos $(23,6 \%)$ componentes de SM. Los más frecuentes fueron niveles bajos de C-HDL $(60,6 \%)$ y la hipertrigliceridemia $(29,9 \%)$, mientras que la obesidad abdominal, HTA e hiperglicemia no sobrepasaron el 3\%. Este fenotipo también ha sido observado en universitarios de Brasil, donde la prevalencia de SM es baja y la mayoría presenta uno o dos componentes aislados, siendo el componente más frecuente el bajo nivel de C-HDL, y el menos frecuente la hiperglicemia ${ }^{(27,28)}$. En nuestro estudio, el punto de corte de la glicemia en ayunas $(\geq 110$ $\mathrm{mg} / \mathrm{dL}$ ) puede ser el determinante de la baja frecuencia del componente en mujeres y de su ausencia en varones universitarios, sin embargo, incluso ajustando el corte a $100 \mathrm{mg} / \mathrm{dL}$, la hiperglicemia en universitarios no variaría debido a que la glicemia en este grupo oscila en $\pm 6,9$ desviaciones estándar alrededor de $86,9 \mathrm{mg} /$ dL. Esta explicación es consistente con lo encontrado en universitarios del Ecuador, donde se encontró una prevalencia de hiperglicemia del $0,2 \%$ en mujeres y de $0,3 \%$ en varones (punto de corte $100 \mathrm{mg} / \mathrm{dL}$ ) ${ }^{(10)}$. 
Tabla 5. Características asociadas a síndrome metabólico en estudiantes de educación secundaria, universitarios y madres de estudiantes de primaria, región Cajamarca, Perú 2014

\begin{tabular}{|c|c|c|c|c|c|c|c|c|c|}
\hline \multirow[t]{2}{*}{ Variable } & \multicolumn{3}{|c|}{ Estudiantes educación secundaria } & \multicolumn{3}{|c|}{ Estudiantes universitarios } & \multicolumn{3}{|c|}{ Madres de estudiantes de primaria } \\
\hline & n (\%) & OR (IC95\%) & valor $p$ & $\%$ & OR (IC95\%) & valor $p$ & n (\%) & OR (IC95\%) & valor $p$ \\
\hline$\overline{S e x o}$ & & & 0,177 & & & $0,029^{\star}$ & & & na \\
\hline Masculino & $12(4,3)$ & 1,00 & & $0(0,0)$ & & & - & & \\
\hline Femenino & $7(2,3)$ & $0,53(0,20-1,36)$ & & $5(3,3)$ & $\mathrm{nc}$ & & $126(23,5)$ & & \\
\hline Antecedente de DM & & & $0,040^{*}$ & & & $0,428^{*}$ & & & 0,169 \\
\hline No & $15(2,8)$ & 1,00 & & $4(1,5)$ & 1,00 & & $115(22,9)$ & 1,00 & \\
\hline Sí & $4(9,5)$ & $3,71(1,17-11,74)$ & & $1(3,1)$ & $2,17(0,24-20,03)$ & & $11(33,3)$ & $1,69(0,79-3,58)$ & \\
\hline Antecedente de HTA & & & $0,312^{*}$ & & & $0,255^{*}$ & & & 0,822 \\
\hline No & $15(3,0)$ & 1,00 & & $3(1,2)$ & 1,00 & & $89(23,2)$ & 1,00 & \\
\hline Sí & $4(5,0)$ & $1,72(0,56-5,33)$ & & $2(3,3)$ & $2,78(0,45-17,03)$ & & $37(24,2)$ & $1,05(0,68-1,64)$ & \\
\hline Antecedente de ACV & & & $0,394^{*}$ & & & $1,000^{*}$ & & & 0,456 \\
\hline No & $18(3,2)$ & 1,00 & & $5(1,8)$ & $\mathrm{nc}$ & & $120(23,9)$ & $0,71(0,29-1,76)$ & \\
\hline Sí & $1(6,7)$ & $2,19(0,27-17,61)$ & & $0(0,0)$ & & & $6(18,2)$ & 1,00 & \\
\hline Consumo de cigarros & & & $0,426^{*}$ & & & $1,000^{*}$ & & & 0,772 \\
\hline No & $16(3,0)$ & 1,00 & & $3(1,8)$ & $0,77(0,13-4,69)$ & & $111(23,3)$ & 1,00 & \\
\hline Sí & $3(5,1)$ & $1,71(0,48-6,05)$ & & $2(1,4)$ & 1,00 & & $15(25,0)$ & $1,09(0,59-2,04)$ & \\
\hline Consumo de alcohol & & & 0,234 & & & $1,000^{*}$ & & & 0,971 \\
\hline No & $8(2,5)$ & 1,00 & & $0(0,0)$ & $\mathrm{nc}$ & & $25(23,6)$ & $0,99(0,60-1,64)$ & \\
\hline Sí & $11(4,2)$ & $1,74(0,69-4,40)$ & & $5(1,8)$ & & & $100(23,4)$ & 1,00 & \\
\hline Consumo de gaseose & & & 0,392 & & & $0,161^{*}$ & & & 0,091 \\
\hline Sí & $14(2,9)$ & 1,00 & & $5(2,6)$ & & & $70(21,1)$ & 1,00 & \\
\hline No & $5(4,5)$ & $1,57(0,55-4,46)$ & & $0(0,0)$ & $\mathrm{nc}$ & & $56(27,5)$ & $0,58(0,42-0,83)$ & \\
\hline Consumo de snacks & & & $0,234^{*}$ & & & $1,000^{*}$ & & & 0,982 \\
\hline Sí & $16(3,0)$ & 1,00 & & $4(1,7)$ & $1,31(0,14-11,90)$ & & $57(23,6)$ & 1,00 & \\
\hline No & $3(5,8)$ & $0,50(0,14-1,79)$ & & $1(1,3)$ & 1,00 & & $69(23,5)$ & $1,00(0,67-1,50)$ & \\
\hline Consumo apropiado & de agua $(\geq$ & .5vasos/día) & 0,029 & & & $1,000^{*}$ & & & 0,148 \\
\hline No & $12(2,5)$ & 1,00 & & $4(1,7)$ & 1,00 & & $111(24,7)$ & $0,65(0,35-1,17)$ & \\
\hline Sí & $7(6,7)$ & $2,79(1,07-7,27)$ & & $1(1,5)$ & $0,87(0,09-7,91)$ & & $15(17,4)$ & 1,00 & \\
\hline Consumo adecuado & de fruta & & 0,934 & & & $1,000^{*}$ & & & 0,55 \\
\hline No & $6(3,3)$ & 1,00 & & $2(1,7)$ & 1,00 & & $44(22,1)$ & 1,00 & \\
\hline Sí & $13(3,2)$ & $0,96(0,36-2,57)$ & & $3(1,6)$ & $0,91(0,15-5,54)$ & & $82(24,4)$ & $1,13(0,75-1,73)$ & \\
\hline Consumo adecuado & de verdur & & 0,638 & & & $1,000^{*}$ & & & 0,006 \\
\hline No & $13(3,5)$ & 1,00 & & $3(1,9)$ & 1,00 & & $65(29,5)$ & 1,00 & \\
\hline Sí & $6(2,8)$ & $0,79(0,29-2,11)$ & & $2(1,4)$ & $0,72(0,12-4,36)$ & & $61(19,3)$ & $0,57(0,38-0,85)$ & \\
\hline Actividad física & & & $0,087^{\star}$ & & & $0,635^{*}$ & & & 0,044 \\
\hline Baja & $16(4,3)$ & 1,00 & & $3(1,4)$ & 1,00 & & $94(26,2)$ & 1,00 & \\
\hline $\begin{array}{l}\text { Moderada o } \\
\text { alta }\end{array}$ & $3(1,4)$ & $0,32(0,09-1,12)$ & & $2(2,2)$ & $1,59(0,26-9,73)$ & & $32(18,3)$ & $0,63(0,40-0.99)$ & \\
\hline Estado nutricional & & & & & & & & & \\
\hline $\begin{array}{l}\text { No sobrepeso, } \\
\text { ni obesidad }\end{array}$ & $1(0,2)$ & 1 & $<0,001^{*}$ & $0(0)$ & $\mathrm{nc}$ & $0,001^{*}$ & $10(6,8)$ & 1 & $<0,001$ \\
\hline $\begin{array}{l}\text { Exceso de } \\
\text { peso }\end{array}$ & $18(10,3)$ & $47,42(6,28-358,24)$ & & $5(6,1)$ & & & $116(29,8)$ & $5,82(2,96-11,46)$ & \\
\hline
\end{tabular}

*Prueba exacta de Fisher

nc: OR crudo no calculado, una celda con valor observado de 0

na: no aplica

DM: diabetes mellitus, HTA: hipertensión arterial, ACV: accidente cerebrovascular 
Con respecto a los estudiantes de secundaria, nuestro hallazgo $(3,2 \%)$ es levemente superior a lo reportado entre el 2013-2014 en adolescentes (12 a 17 años) de Brasil, en quienes se encontró una frecuencia del 2,6\% ${ }^{(29)}$. Estudios realizados en adolescentes de Venezuela y Colombia encontraron prevalencias de $2,2{ }^{(7)}$ y $2,5 \%$ (30), respectivamente. Al igual que en universitarios, la hiperglicemia es poco frecuente, mientras que la hipertrigliceridemia y el bajo nivel de C-HDL se presentan en una importante proporción, pero en forma aislada. La baja frecuencia de hiperglicemia en adolescentes, ha sido reportada en los Estados Unidos (punto de corte para glicemia $=110 \mathrm{mg} / \mathrm{dL}$ ) ${ }^{(31)}$, mientras que en adolescentes de Brasil ${ }^{(29)}$ y Venezuela ${ }^{(7)}$ usando un corte de $100 \mathrm{mg} / \mathrm{L}$ la prevalencia de hiperglicemia fue ligeramente mayor (4,1 y 3,6\% respectivamente); en los estudios referidos la hiperglicemia fue el componente de SM menos frecuente ${ }^{(7,29,30)}$.

Los componentes más frecuentes de SM fueron la hipertrigliceridemia y el bajo nivel de C-HDL, dicha combinación se presenta en los tres grupos de estudio. La hipertrigliceridemia se encuentra en $46,4 \%$ de estudiantes de secundaria, este porcentaje podría explicarse por el punto de corte utilizado ( $\geq 110 \mathrm{mg} / \mathrm{dL})$, el cual es menor comparado con el corte para adultos ( $\geq 150 \mathrm{mg} / \mathrm{dL}$ ). La prevalencia de hipertrigliceridemia encontrada en universitarios $(29,9 \%)$ y madres $(38,4 \%)$, es similar a lo encontrado en otros estudios realizados en población latinoamericana (2), incluyendo a lo reportado en Arequipa (52\% en varones y $35,3 \%$ en mujeres) $)^{(3)}$; pero inferior a lo reportado para mujeres de Lima $(61,5 \%)^{(6)}$.

El bajo nivel de C-HDL es el componente más frecuente en nuestro estudio $(60,5 \%$ en universitarios y $72,3 \%$ en madres de primaria). Este componente también ha sido el más frecuente en madres de las ciudades de Trujillo, Arequipa, Callao y Lima $(50,1 \%)^{(4)}$ y mujeres adultas de Arequipa $(60,9 \%)^{(3)}$. El estudio MIGRANT ${ }^{(32)}$ realizado en población rural de Ayacucho reporta una elevada prevalencia de niveles bajos de C-HDL $(56,2 \%)$, cabe destacar que en dicho estudio la prevalencia llegó a $75,5 \%$ en el grupo de las mujeres, magnitud comparable a lo encontrado en el grupo de madres de nuestro estudio.

La hiperglicemia es el componente menos frecuente, solo el $1,4 \%$ de las madres de primaria lo presentaron. Su ocurrencia es marcadamente inferior a lo encontrada en otros estudios ${ }^{(2,5)}$. A pesar que la hiperglicemia también ha sido reportada como el componente menos frecuente en el estudio PREVENCION, esta llega a $5,4 \%$ en varones y $5,0 \%$ en mujeres ${ }^{(3)}$. En el estudio CARMELA realizado en la ciudad de Lima, se encontró un 30,7\% de hiperglicemia en varones y $24,0 \%$ en mujeres ${ }^{(6)}$, estas magnitudes encontradas en la capital del Perú, son más próximas a lo reportado en población adulta de los Estados Unidos $(19,9 \%)^{(33)}$, y en Colombia $(19,8 \%)^{(34)}$.
La obesidad abdominal se presenta principalmente en el grupo de madres, con magnitudes superiores a lo encontrado en adultos de ciudades andinas (Arequipa, Perú con 36,9\%; y Zulia, Venezuela con 42,9\%) ${ }^{(3,35)}$, y en mujeres peruanas residentes por encima de los $3000 \mathrm{~m}$ de altitud $(37,5 \%)^{(25)}$. La obesidad abdominal en el grupo de madres se aproxima a lo encontrado en los Estados Unidos, donde en adultos se ha mantenido alrededor del $50 \%$ desde 1999 , y en el periodo 2009 2010 dicha prevalencia fue de $46,4 \%$ en varones y $65,4 \%$ en mujeres ${ }^{(33)}$. Por otro lado, la magnitud de obesidad abdominal en adolescentes de nuestro estudio (5,6\%), es inferior a lo encontrado en adolescentes españoles de 12 a 17 años en quienes se reporta un $11,6 \%{ }^{(36)}$, y en adolescentes norteamericanos de 12 a 18 años en quienes la prevalencia (periodo 2011-2012) fue $22,4 \%$ (37).

En los tres grupos se encontró que el exceso de peso (sobrepeso/obesidad) estuvo asociado con SM. La obesidad como factor de riesgo de SM en adolescentes y adultos jóvenes ha sido reportada en adolescentes de Argelia ${ }^{(19)}$, niños mexicanos ${ }^{(38)}$, y en niños y adultos jóvenes (3 a 19 años) de los Estados Unidos ${ }^{(39)}$. En nuestro estudio, la fuerza de asociación entre exceso de peso y SM fue mayor en los estudiantes de secundaria (OR crudo $=47,42)$, comparada con la encontrada en madres de primaria $(\mathrm{OR}$ crudo $=5,82)$. Estos resultados sugerirían que la obesidad en adolescentes está asociada con mayor riesgo de SM; por ello, la identificación del exceso de peso en niños y adolescentes se constituiría en una buena estrategia para reducir el riesgo de SM en etapas posteriores de la vida ${ }^{(40)}$. Respecto al nivel de actividad física, encontramos en el grupo de madres, que realizar niveles moderados o altos de actividad física es un factor protector para el desarrollo de SM. En estudiantes de secundaria se encontró una tendencia como factor protector, pero no fue significativa; en universitarios no se encontró dicha asociación, lo cual podría ser explicado por la forma de medición del nivel de actividad física (autorreporte), y el limitado número de casos observados de SM en universitarios.

Las asociaciones del SM con las prácticas saludables $\mathrm{y}$ hábitos nocivos tuvieron un comportamiento diferente entre los grupos estudiados. En universitarios y estudiantes de secundaria no se encontraron asociaciones significativas del SM con antecedentes patológicos (DM o HTA), consumo de cigarros, bebidas gasificadas, snacks, consumo de fruta y verduras, esto podría ser explicado por la baja frecuencia de SM, especialmente en universitarios, lo cual pudo afectar la potencia estadística, sobre todo en este grupo de estudio.

Nuestros resultados brindan evidencia adicional a la descripción del patrón fenotípico de SM en población andina. En mujeres adultas (grupo de madres), dicho patrón se caracterizó por una alta frecuencia de obesidad abdominal y de dislipidemias (hipertrigliceridemia y niveles bajo de C-HDL). En adolescentes de educación secundaria 
y adultos jóvenes universitarios el SM se caracteriza por la elevada frecuencia de dislipidemias, siendo menos frecuente la obesidad abdominal, las alteraciones de glicemia y presión arterial. Además, se observa incremento en la prevalencia de SM según grupo etario, siendo este incremento más pronunciado en mujeres ${ }^{(3,5)}$

Entre las limitaciones del estudio, tenemos el diseño transversal que no permite establecer una relación de causalidad entre el estado nutricional y el SM, sin embargo, dicha asociación se presenta en todos los grupos de estudio, presenta una fuerte fuerza de asociación y es consistente con lo reportado en otros estudios. Si bien la selección de participantes fue realizada de manera probabilística, el diseño muestral solo es representativo para las poblaciones estudiadas, y no pretenden ser estimaciones para la población de adolescentes, adultos jóvenes y mujeres de la región Cajamarca, $\mathrm{u}$ otras poblaciones andinas. Las poblaciones de estudio fueron seleccionadas en centros educativos y una universidad, instituciones que agrupan individuos con características socioeconómicas similares; y cuyo entorno institucional ofrece mayores posibilidades de implementar intervenciones para reducir la obesidad/sobrepeso.

En conclusión, la frecuencia de SM se presenta en diferentes magnitudes según grupo de estudio, siendo las madres de estudiantes de primaria el grupo más afectado. La magnitud en escolares adolescentes y universitarios es mucho menor, y su frecuencia es comparable con estudios realizados en poblaciones de diferentes características socioculturales y ambientales. El exceso de peso es la principal condición modificable para SM, y su fuerza de asociación es mayor en estudiantes de secundaria. Esto implicaría evidencia para focalizar intervenciones dirigidas a reducir la obesidad y el sobrepeso, en las escuelas y centros universitarios. Futuros estudios epidemiológicos que incluyan determinación de los factores genéticos más importantes permitirían explicar la elevada prevalencia de las dislipidemias, las cuales se presentan desde la adolescencia.

Agradecimientos: a Martha Rodríguez Tejada e Yrene Yupanqui Vásquez quienes brindaron apoyo en la concepción y el diseño del estudio. A Rosa Chávez Farro y Dora Rabanal Pita quienes apoyaron en concepción y en la recolección de datos. A Daysi Ninatanta Ortiz quien también apoyó en la recolección de datos.

Contribuciones de autoría: JNO, LNZ, AGF participaron en la concepción, diseño del estudio, recolección de datos, y construcción de la base de datos. FRR participó en la concepción y diseño del estudio. Todos los autores participaron en el análisis e interpretación de los datos, redacción del manuscrito, revisión crítica y su aprobación final.

Fuente de financiamiento: la investigación fue financiada por la Universidad Nacional de Cajamarca, según Resolución Rectoral 038-2013-UNC, de fecha 27 de febrero de 2013, donde se resuelve "aprobar los proyectos de investigación, presentados por los docentes de la Universidad Nacional ce Cajamarca para ser financiados con Fondos Concursables Provenientes del Canon y Minero, Sobrecanon y Regalías Mineras, correspondientes a la convocatoria 2012, Financiamiento de investigación Docente Canon Minero".

Conflictos de interés. los autores declaran no tener conflictos de interés.

\section{REFERENCIAS BIBLIOGRÁFICAS}

1. Grundy SM. Metabolic syndrome update. Trends Cardiovasc Med. 2016;26(4):36473. doi: $10.1016 /$ j.tcm.2015.10.004

2. Márquez-Sandoval F, Macedo-Ojeda G, Viramontes-Hörner D, Fernández Ballart J, Salas Salvadó J, Vizmanos B. The prevalence of metabolic syndrome in Latin America: a systematic review. Public Health Nutr. 2011; 14(10):1702-13. doi: $10.1017 /$ S1368980010003320.

3. Medina-Lezama J, Zea-Diaz H, MoreyVargas OL, Bolaños-Salazar JF, MuñozAtahualpa E, Postigo-MacDowall M, et al. Prevalence of the metabolic syndrome in Peruvian Andean hispanics: The PREVENCION study. Diabetes Res Clin Pract. 2007; 78(2):270-81. DOI: 10.1016/j.diabres.2007.04.004

4. Núñez-Robles E, Huapaya-Pizarro C, Torres-Lao R, Esquivel-León S, SuarezMoreno V, Yasuda-Espinoza M, et al. Prevalence of cardiovascular and metabolic risk factors in school students, university students, and women from community-based organizations in the districts of Lima, Callao, la Libertad and Arequipa, Peru 2011. Rev Peru Med Exp
Salud Pública. 2014; 31(4):652-659.

5. Chirinos DA, Morey-Vargas OL, Goldberg RB, Chirinos JA, MedinaLezama J. Metabolic syndrome in Andean populations. Glob Heart. 2013; 8(4):349354. doi: 10.1016/j.gheart.2013.10.001.

6. Escobedo J, Schargrodsky $\mathrm{H}$, Champagne B, Silva $\mathrm{H}$, Boissonnet CP, Vinueza $\mathrm{R}$, et al. Prevalence of the Metabolic Syndrome in Latin America and its association with sub-clinical carotid atherosclerosis: the CARMELA cross sectional study. Cardiovasc Diabetol. 2009; 8(1):52. doi: 10.1186/1475-2840-8-52

7. Reyes MV, Mederico M, de Valeri MP, Briceño Y, Zerpa Y, Gómez-Pérez R, et al. Síndrome metabólico en escolares y adolescentes de la ciudad de MéridaVenezuela: comparación de resultados utilizando valores de referencia locales e internacionales (estudio CREDEFAR). Endocrinol Nutr. 2014; 61(9):474-485. doi.org/10.1016/j.endonu.2014.03.009

8. Lim S, Jang HC, Park KS, Cho SI, Lee M-G, Joung $\mathrm{H}$, et al. Changes in metabolic syndrome in American and Korean youth, 1997-2008. Pediatrics.
2013; 131(1):e214-222. doi: 10.1542/ peds.2012-0761

9. Feliciano-Alfonso JE, Mendivil CO, Ariza IDS, Pérez CE. Cardiovascular risk factors and metabolic syndrome in a population of young students from the National University of Colombia. Rev Assoc Médica Bras. 2010; 56(3):293-298.

10. Nieto CIR, Pérez JDM, Freire LM, Morales KRDP, Romero CVE. Prevalencia de síndrome metabólico y factores de riesgo asociados en jóvenes universitarios ecuatorianos. Nutr Hosp. 2015; 31(n04):1574-1581.

11. Khan RJ, Gebreab SY, Riestra P, Xu R, Davis SK. Parent-offspring association of metabolic syndrome in the Framingham Heart Study. Diabetol Metab Syndr. 2014; 6(1):140. doi: 10.1186/1758-5996-6140.

12. Rosini N, Oppermann S, Diegoli R, Machado M, da Silva E. Metabolic Syndrome and Importance of Associated Variables in Children and Adolescents in Guabiruba - SC, Brazil. Arq Bras Cardiol. 2015; 105(1):37-44. doi.org/10.5935/ abc. 20150040 
13. Programa de familias y viviendas saludables. Ministerio de Salud. Dirección General de Promoción de la Salud. Lima. Ministerio de Salud; 2006.

14. Lineamientos de Política y Estrategias para la Prevención y Control de Enfermedades No Transmisibles (ENT) 2016-2020. Resolución Ministerial N²29-2016/ MINSA.

15. Pedroza-Tobias A, Trejo-Valdivia B, Sanchez-Romero LM, Barquera S. Classification of metabolic syndrome according to lipid alterations: analysis from the Mexican National Health and Nutrition Survey 2006. BMC Public Health. 2014; 14(1):1. DOI: $10.1186 / 1471-2458-14-1056$

16. Khosravi-Boroujeni $\mathrm{H}$, Ahmed $\mathrm{F}$, Sadeghi M, Roohafza H, Talaei M, Dianatkhah M, et al. Does the impact of metabolic syndrome on cardiovascular events vary by using different definitions? BMC Public Health. 2015; 15(1). doi: 10.1186/s12889-015-2623-3

17. Grundy SM, Cleeman JI, Daniels SR, Donato KA, Eckel RH, Franklin BA, et al. Diagnosis and Management of the Metabolic Syndrome. Circulation. 2005; 112(17): 2735-52. dx.doi.org/10.1161/ CIRCULATIONAHA.105.169404

18. Cook S, Weitzman M, Auinger P, Nguyen M, Dietz WH. Prevalence of a metabolic syndrome phenotype in adolescents: Findings from the third national health and nutrition examination survey, 1988-1994. Arch Pediatr Adolesc Med. 2003; 157(8):821-7. DOI: 10.1001/ archpedi.157.8.821

19. Benmohammed K, Valensi P, Benlatreche M, Nguyen MT, Benmohammed F, Pariès J, et al. Anthropometric markers for detection of the metabolic syndrome in adolescents. Diabetes Metab. 2015; 41(2):138-44. doi: 10.1016/j. diabet.2014.07.001.

20. Sangun Ö, Dündar B, Köşker $M$, Pirgon Ö, Dündar N. Prevalence of Metabolic Syndrome in Obese Children and Adolescents using Three Different Criteria and Evaluation of Risk Factors. J Clin Res Pediatr Endocrinol. 2011; 3(2):70-6. doi: 10.4274/jcrpe.v3i2.15.

21. Guías para el Procesamiento de Datos y Análisis del Cuestionario Internacional de Actividad Física (IPAQ) - Versiones Corta y Larga. Universidad de Granada. Junta de Andalucía; 2005.

22. Aguilar L, Contreras M, Del CantoJ. Guía Técnica para la valoración nutricional antropométrica de la persona adulta. Ministerio de Salud, Instituto Nacional de Salud. 2012 Disponible en: http://www. ins.gob.pe/repositorioaps/0/5/jer/otros lamejo_cenan/Gu\%C3\%ADa\%20
T\%C3\%A9cnica\%20VNA\%20Adulto. pdf

23. Aguilar L, Contreras M, Calle M del C. Guía técnica para la valoración nutricional antropométrica de la persona adolescente. Ministerio de Salud, Instituto Nacional de Salud. 2015. Disponible en: http:// repositorio.ins.gob.pe/bitstream/ INS/214/1/CENAN-0056.pdf

24. Guía Técnica de Práctica Clínica para la Prevención y Control de la Enfermedad Hipertensiva en el Primer Nivel de Atención. Resolución Ministerial N ${ }^{\circ} 031$ 2015/MINSA.

25. Pajuelo J, Sanchez J, Torres H, Miranda M. Prevalencia del síndrome metabólico en pobladores peruanos por debajo de 1000 y por encima de los $3000 \mathrm{msnm}$. An Fac med. 2012; 73(2):101-6.

26. Martínez MA, Leiva AM, Sotomayor C, Victoriano T, Von Chrismar P, Pineda S. Factores de riesgo cardiovascular en estudiantes de la Universidad Austral de Chile. Rev Médica Chile. 2012; 140(4):426-435. dx.doi.org/10.4067/ S0034-98872012000400002

27. Silva ARV da, Sousa LSN de, Rocha T de S, Cortez RMA, Macêdo LG do N, Almeida PC de. Prevalence of metabolic components in university students. Rev Lat Am Enfermagem. 2014; 22(6):1041-7.dx.doi.org/10.1590/01041169.0129 .2514

28. deFreitas RWF, de Araújo MFM, Marinho NBP, de Vasconcelos HCA, Lima ACS, Pereira DCR, et al. Prevalence of the metabolic syndrome and its individual components in Brazilian college students. J Clin Nurs. 2013; 22(9-10):1291-8. doi: 10.1111/jocn.12015.

29. Kuschnir MCC, Bloch KV, Szklo M, Klein CH, Barufaldi LA, Abreu G de A, et al. ERICA: prevalence of metabolic syndrome in Brazilian adolescents. Rev Saude Publica. 2016; 50 Suppl 1:11s. doi: 10.1590/S01518-8787.2016050006701.

30. Suárez-Ortegón MF, Ramírez-Vélez R, Mosquera M, Méndez F, Aguilar-de Plata C. Prevalence of metabolic syndrome in urban Colombian adolescents aged 1016 years using three different pediatric definitions. J Trop Pediatr. 2013; 59(2):145-9.

31. de Ferranti SD, Gauvreau K, Ludwig DS, Neufeld EJ, Newburger JW, Rifai N. Prevalence of the Metabolic Syndrome in American Adolescents: Findings From the Third National Health and Nutrition Examination Survey. Circulation. 2004; 110(16):2494-7. DOI: 10.1161/01. CIR.0000145117.40114.C7

32. Lazo-Porras M, Bernabe-Ortiz A, Málaga G, Gilman RH, Acuña-Villaorduña A, Cardenas-Montero D, et al. Low HDL cholesterol as a cardiovascular risk factor in rural, urban, and rural-urban migrants: PERU MIGRANT cohort study. Atherosclerosis. 2016; 246:36-43. DOI: 10.1016/j.atherosclerosis.2015.12.039

33. Beltrán-Sánchez $\mathrm{H}$, Harhay MO, Harhay MM, McElligott $S$. Prevalence and Trends of Metabolic Syndrome in the Adult U.S. Population, 1999-2010. J Am Coll Cardiol. 2013; 62(8):697-703. doi: 10.1016/j.jacc.2013.05.064.

34. Davila EP, Quintero MA, Orrego ML, Ford ES, Walke H, Arenas MM, et al. Prevalence and risk factors for metabolic syndrome in Medellin and surrounding municipalities, Colombia, 2008-2010. Prev Med. 2013; 56(1):30-4. doi: 10.1016/j.ypmed.2012.10.027.

35. Florez $\mathrm{H}$, Silva E, Fernández V, Ryder E, Sulbarán T, Campos G, et al. Prevalence and risk factors associated with the metabolic syndrome and dyslipidemia in White, Black, Amerindian and Mixed Hispanics in Zulia State, Venezuela. Diabetes Res Clin Pract. 2005; 69(1):6377. DOI: 10.1016/j.diabres.2004.11.018

36. Schröder H, Ribas L, Koebnick C, Funtikova A, Gomez SF, Fíto $M$, et al. Prevalence of Abdominal Obesity in Spanish Children and Adolescents. Do We Need Waist Circumference Measurements in Pediatric Practice? PLoS ONE.2014; 9(1): e87549. doi: 10.1371/ journal.pone.0087549

37. Xi B, Mi J, Zhao M, Zhang T, Jia C, Li J, et al. Trends in Abdominal Obesity Among US Children and Adolescents. Pediatrics. 2014; 134(2):e334-9. doi: 10.1542/ peds.2014-0970

38. Guzman I, Salgado A, Muñoz J, Vences A, Parra I. Prevalence of metabolic syndrome in children with and without obesity. Med Clin (Barc). 2015; 144(5):198-203. doi: 10.1016/j.medcli.2013.10.033.

39. Skinner AC, Perrin EM, Moss LA, Skelton JA. Cardiometabolic Risks and Severity of Obesity in Children and Young Adults. N Engl J Med. 2015; 373(14):1307-17. DOI: 10.1056/NEJMoa1502821

40. Kelishadi R, Gheiratmand R, Ardalan G, Adeli K, Mehdi Gouya M, Mohammad Razaghi E, et al. Association of anthropometric indices with cardiovascular disease risk factors among children and adolescents: CASPIAN Study. Int J Cardiol. 2007; 117(3):340-8. DOI: $10.1016 /$ j. ijcard.2006.06.012

Correspondencia: Juana Ninatanta Ortiz Dirección: Jr. Cinco Esquinas 1590 Cajamarca, Perú

Teléfono: (076) 976111415

Correoelectrónico:jano_unc@hotmail.com 